\title{
Heterogeneity of Skin Surface Oxygen Level of Wrist in Relation to Acupuncture Point
}

\author{
Minyoung Hong, ${ }^{1}$ Sarah S. Park, ${ }^{2}$ Yejin Ha, ${ }^{2}$ Jaegeun Lee, ${ }^{1}$ Kwangsik Yoo, ${ }^{1}$ \\ Gil-Ja Jhon, ${ }^{2}$ Minah Suh, ${ }^{1,3}$ and Youngmi Lee ${ }^{2}$ \\ ${ }^{1}$ Department of Biological Science, Sungkyunkwan University, Suwon 440-746, Republic of Korea \\ ${ }^{2}$ Department of Chemistry and Nano Science, Ewha Womans University, Seoul 120-750, Republic of Korea \\ ${ }^{3}$ Graduate Program for Health Science and Technology, Sungkyunkwan University, Suwon 440-746, Republic of Korea
}

Correspondence should be addressed to

Minah Suh, minahsuh@skku.edu and Youngmi Lee, youngmilee@ewha.ac.kr

Received 28 February 2012; Accepted 13 March 2012

Academic Editor: Gerhard Litscher

Copyright () 2012 Minyoung Hong et al. This is an open access article distributed under the Creative Commons Attribution License, which permits unrestricted use, distribution, and reproduction in any medium, provided the original work is properly cited.

The distribution of partial oxygen pressure $\left(\mathrm{pO}_{2}\right)$ is analyzed for the anterior aspect of the left wrist with an amperometric oxygen microsensor composed of a small planar Pt disk-sensing area (diameter $=25 \mu \mathrm{m}$ ). The $\mathrm{pO}_{2}$ levels vary depending on the measurement location over the wrist skin, and they are systematically monitored in the analysis for both one-dimensional single line (along the wrist transverse crease) and two-dimensional square area of the wrist region. Relatively higher $\mathrm{pO}_{2}$ values are observed at certain area in close proximity to the position of acupuncture points with statistical significance, indicating strong relationship between oxygen and acupuncture point. The used oxygen microsensor is sensitive enough to detect the $\mathrm{pO}_{2}$ variation depending on the location. This study may provide information helpful to understand possible physiological roles of the acupuncture points.

\section{Introduction}

Acupuncture is a method of medical treatments, based on inserting small needles on the specified body skin locations called acupuncture points. The practice of acupuncture as a healing treatment dates back over 2500 years in traditional eastern medicine. In the late 20th century, acupuncture became to be accepted as an alternative and complementary therapy even in western countries including the United States [1]. In fact, the National Institute of Health (NIH) published a consensus on the use acupuncture in the treatment of pain symptom in 1997 [2].

According to acupuncture meridian theory, a network of 12 main meridians passes through body internal organs and links acupuncture points on skin together. Through the meridian channels, a vital energy, called Qi, circulates the body to regulate body functions. Acupuncture is considered to stimulate the Qi circulation to attain the balance of Qi. Although acupuncture practice is widely used for chronic illness, the efficacy and mechanism of the acupuncture action in mediating analgesia still remain controversial. Indeed, the lack of anatomical and scientific evidence supporting the existence of meridians, acupuncture points, and Qi makes more difficult for the acupuncture treatment to be generally accepted in modern science. Some research efforts for anatomical studies of meridians and acupuncture points have been reported [3-11]. However, there is still controversy since the experimental results could not provide direct/ obvious evidence, and moreover they often show a lack of reproducibility.

In acupuncture studies, three kinds of Qi are described to be obtained from air, food, and inheritance, suggesting the close relationship between Qi and air (i.e., oxygen) [1]. Oxygen is essential for energy metabolism in most living organisms. Meanwhile, higher expression of nitric oxide (NO) synthase enzyme, producing endogenous NO, was reported around skin acupuncture points and meridians than other areas [12]. NO is a well-known vasodilator increasing 
blood flow and volume and therefore relates to oxygen transport in body [13]. From these separate reports, we inferred that acupuncture points were possibly associated with body oxygen supply and, therefore, recently reported the real-time quantitative measurements of oxygen levels on acupuncture points using a highly sensitive electrochemical oxygen microsensor [14]. The localized oxygen levels at small acupuncture points and at nearby nonacupuncture points were measured successfully, because of the small planar dimension of the sensor (sensing diameter $=25 \mu \mathrm{m}$ ). In fact, the oxygen levels measured at two acupuncture points (LI4 (Hegu) and PC8 (Laogong)) were observed to be higher than those at the corresponding nonacupuncture points, providing an evidence of the physical existence of acupuncture points which may be functionally connected with the oxygen supply [14]. Advanced from the previous work, this paper reports the blind measurements of oxygen levels within confined areas of wrist skin surface and the relationship between the oxygen levels with acupuncture points.

\section{Materials and Methods}

2.1. Electrochemical Oxygen Microsensor. A clark-type amperometric microsensor for selective oxygen measurement was fabricated as described previously [14]. The oxygen microsensor consists of a glass-sealed $\mathrm{Pt}$ disk cathode $(\mathrm{Pt}$ diameter $=25 \mu \mathrm{m}$, Good Fellow) and a coiled $\mathrm{Ag} / \mathrm{AgCl}$ wire anode $(127-\mu \mathrm{m}$ diameter, A-M Systems) covered with PTFE gas-permeable membrane (W. L. Gore \& Associates, thickness $<19 \mu \mathrm{m}$, porosity $50 \%$, pore size $0.05 \mu \mathrm{m}$ ). The composition of an internal solution, both the cathode and anode, is $30 \mathrm{mM} \mathrm{NaCl}$ and $0.3 \mathrm{mM} \mathrm{HCl}$ in deionized water. The surface of the Pt disk cathode was electrochemically platinized using platinizing solution (YSI Inc., Yellow Springs, $\mathrm{OH})$ to increase the real active surface of the electrode and eventually to enhance the sensor sensitivity to oxygen [15]. A potential of $-0.6 \mathrm{~V}$ (versus $\mathrm{Ag} / \mathrm{AgCl}$ anode) was applied to the Pt cathode where the electrochemical reduction of oxygen occurs favorably at this potential. The current between the cathode and anode, induced by the oxygen reduction, was monitored as a function of time using CHI1000A electrochemical analyzer ( $\mathrm{CH}$ Instruments Inc., USA). Asprepared oxygen microsensor was calibrated before and after oxygen measurements by recording the sensor current at $-0.6 \mathrm{~V}$ with successive several injections of a given amount of phosphate-buffered saline (PBS, pH 7.4, Fisher Scientific) solution saturated with oxygen into deaerated PBS (pH 7.4) solution to alter the oxygen concentrations.

2.2. Oxygen Measurements on Wrist Skin. The experimental details for the measurements of oxygen levels on skin are described previously [14]. Briefly, the prepared oxygen microsensor was positioned above the first wrist skin point of interest, which was wetted with a drop of PBS ( $\mathrm{pH}=$ 7.4) solution $(15 \mu \mathrm{L})$. A micromanipulator (World Precision Instrumentation Inc., Sarasota, FL, USA) was used to position the sensor and maintain the separation between the sensor and skin surface, $\sim 1 \mathrm{~mm}$. Then, the sensor current between the cathode and anode, which is proportional to the partial oxygen pressure $\left(\mathrm{pO}_{2}\right)$, was recorded using an electrochemical analyzer. Once the measured current reached to a quite stable one, the sensor was moved horizontally to the second skin point of interest while the sensor current was monitored continuously. After the stable current was achieved at the second point, the sensor was moved to the third point to measure the $\mathrm{pO}_{2}$ level at that location. This whole procedure was repeated until the measurements of $\mathrm{pO}_{2}$ levels for all the projected points were finished.

The measurements of $\mathrm{pO}_{2}$ levels were performed for (1) one-dimensional single line and (2) two-dimensional square area within the wrist independently. For the onedimensional experiment, the sensor currents responding to $\mathrm{pO}_{2}$ levels were measured at 15 different points along the lateral line on the anterior aspect of the left hand-wrist transverse crease. The 15 points were evenly distributed with the same separation $(d=3-3.5 \mathrm{~mm}$ depending on individual subject) between two adjacent points along the transverse wrist crease line. The first point and the last 15 th point were positioned $5 \mathrm{~mm}$ apart from the left and right sides of the wrist as shown in Figure 1(a).

For the two-dimensional analysis, the first 5 points were evenly positioned with the same separation between two adjacent points $(d=10-12 \mathrm{~mm}$ depending on individual subject) along the lateral line on the anterior aspect of the left hand-wrist boundary crease. Again, the first point and the fifth point were positioned $5 \mathrm{~mm}$ apart from the left and right sides, respectively. The central five points (nos. 3, 8, 13, 18 , and 23) were positioned along the centered vertical line dividing the anterior wrist evenly, with the same separation (d) as the one for the first lateral wrist line. Then, the other points could be distributed while keeping the same point-topoint separation as shown in Figure 1(b).

The measurements were carried out for five healthy volunteers (average age $=24.2$ ) in calm and rest conditions at room temperature. None of the subjects were previously treated with acupuncture needle insertion at the skin locations investigated. The measured sensor currents were converted to the corresponding $\mathrm{pO}_{2}$ levels using prior calibration data.

2.3. Data Analysis and Statistics. For each volunteer subject, the $\mathrm{pO}_{2}$ levels measured twice and these two $\mathrm{pO}_{2}$ values obtained at the same location were averaged, and the standard deviation was also calculated independently. The averaged data obtained at the same skin location of five different subjects were also averaged. The data for a few specific points showing relatively higher $\mathrm{pO}_{2}$ values than the other region were compared with that at other points exhibiting relatively lower $\mathrm{pO}_{2}$ values using two tailed $t$-test with a Bonferroni correction. $P$ value $<0.05$ was considered significantly different in statistical meaning.

\section{Results and Discussion}

The analytical performance of an amperometric oxygen microsensor was characterized. Figure 2(a) shows the dynamic 


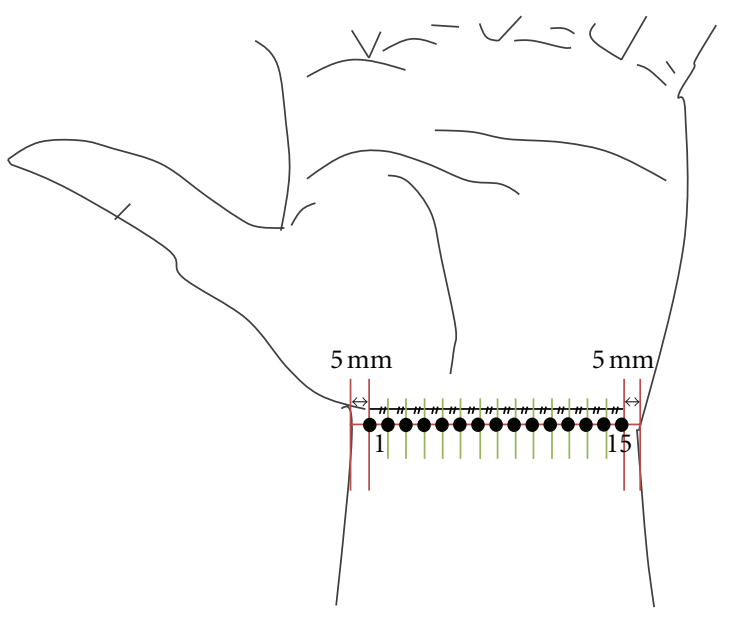

(a)

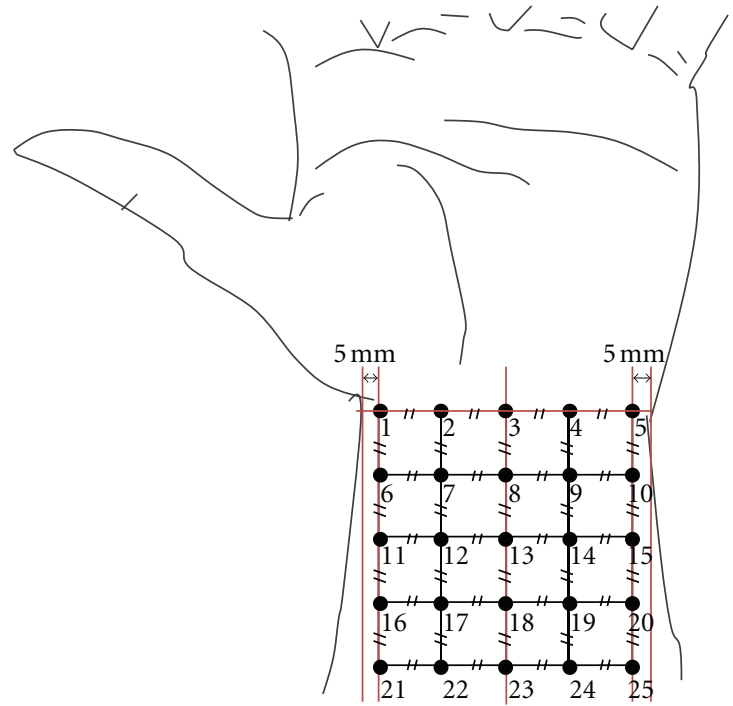

(b)

FIGURE 1: Schematic illustration for the points on the wrist skin where an oxygen microsensor was positioned for $\mathrm{pO}_{2}$ analysis: (a) onedimensional and (b) two-dimensional measurement. The points, No. 1, 15 in (a) and the points, No. 1, 5 in (b) were positioned 5 mm apart from the left and right sides of the wrist. Symbol, //, represents the same separation.

sensor response obtained by measuring the sensor current responding to the $\mathrm{pO}_{2}$ value which was altered by successive injections of a given amount of oxygen standard solution into a deareated PBS sample solution. The sensor current increases in proportion to $\mathrm{pO}_{2}$ value, and the corresponding calibration curve (Figure 2(b)) shows reasonable linearity and sensitivity of $523.8 \pm 58.0 \mathrm{pA} / \mathrm{mmHg}(n=7)$. The sensor sensitivity varied within $<\sim 2 \%$ before and after the skin oxygen measurements and $<0.5 \%$ for $10^{\circ} \mathrm{C}$ temperature change $\left(25-35^{\circ} \mathrm{C}\right)$, confirming the sensor stability.

Figure 3 shows typical data showing the $\mathrm{pO}_{2}$ values monitored at 15 different locations along the transverse wrist crease line. The sensor current measured in terms of time was converted to the $\mathrm{pO}_{2}$ value based on the prior sensor calibration data. Gray-colored sections represent the measurements made over the projected points with the sensorto-skin separation of $\sim 1 \mathrm{~mm}$. Current signals observed in noncolored sections are induced by the sensor movement from one to the other points. In fact, the comparatively higher $\mathrm{pO}_{2}$ values than the other regions are observed at the regions around the points, nos. 1, 8, 9, and 15. For all five different subjects without exception, similar patterns to Figure 3(a) were observed. Figure 3(b) displays the $\mathrm{pO}_{2}$ value averaged for five entire subjects (with standard deviation) corresponding to each point. The $\mathrm{pO}_{2}$ at each point was taken as the average for the data obtained for the last $60 \mathrm{~s}$ of the overall measurement time at that point before the sensor movement to another point. This makes sure that the fully equilibrated $\mathrm{pO}_{2}$ value is obtained. Interestingly, the $\mathrm{pO}_{2}$ values are observed to be closely related to the positions of acupuncture points. In fact, three acupuncture points, LU9 (Taiyuan), PC7 (Daling), and HT7 (Shenmen) from left to right side, are known along the transverse wrist crease line. Rather large standard deviations of the averaged $\mathrm{pO}_{2}$ values could be ascribed to the interindividual variation such as wrist circumference. In the one-dimensional study, the measured $\mathrm{pO}_{2}$ value was in the range of $125-143 \mathrm{mmHg}$ with the greatest difference between the highest and lowest $\mathrm{pO}_{2}$ that was 5.7-9.2 $\mathrm{mmHg}$ depending on the individual subject.

In addition, the $\mathrm{pO}_{2}$ levels at six representative points (nos. 5, 6, 8, 11, 14, and 15) were compared with one another. A two-tailed $t$-test with a Bonferroni correction verifies that the relatively higher $\mathrm{pO}_{2}$ values at the points, nos. 8,14 , and 15 are significantly different from the lower $\mathrm{pO}_{2}$ values at the points, nos. 5, 6, and 11 (Table 1). Current study is in good agreement with our previous work reporting higher $\mathrm{pO}_{2}$ levels at the acupuncture points (LI4 and PC8) than at nonacupuncture points.

As indicated in the Methods section, the $\mathrm{pO}_{2}$ analysis was also carried out at 25 different points evenly distributed in two-dimensional square area over the wrist as depicted in Figure $1(\mathrm{~b})$. The measured $\mathrm{pO}_{2}$ values showed even higher interindividual variation in this two-dimensional analysis compared to the one-dimensional one. It is presumably considered that the subject body size difference induces a relatively large variance in the analysis of a wider region. Therefore, typical measurement examples are presented without the statistical analysis of the overall subjects.

For convenient comparison purpose, each measured $\mathrm{pO}_{2}$ was normalized to the average of all 25 values. In fact, the normalized $\mathrm{pO}_{2}$ was obtained as follows:

$$
\mathrm{pO}_{2, \text { norm }}=\frac{\mathrm{pO}_{2}}{\mathrm{pO}_{2, \mathrm{avg}}},
$$

where $\mathrm{pO}_{2 \text {,norm }}$ is the normalized $\mathrm{pO}_{2} ; \mathrm{pO}_{2}$ is the measured $\mathrm{pO}_{2}$ value at each point; $\mathrm{pO}_{2, \mathrm{avg}}$ is the average of all the $\mathrm{pO}_{2}$ values measured at 25 different point for each subject. 


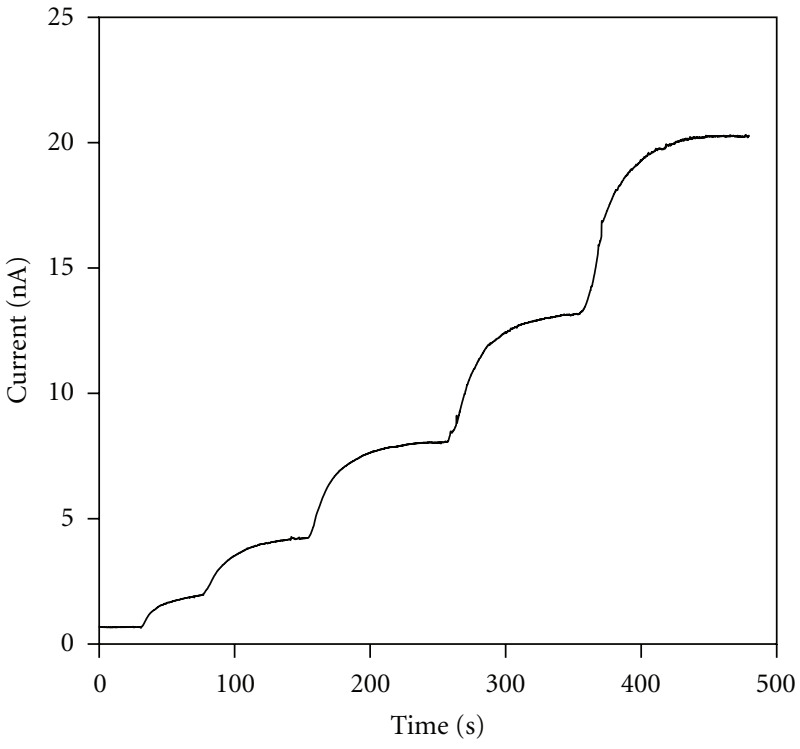

(a)

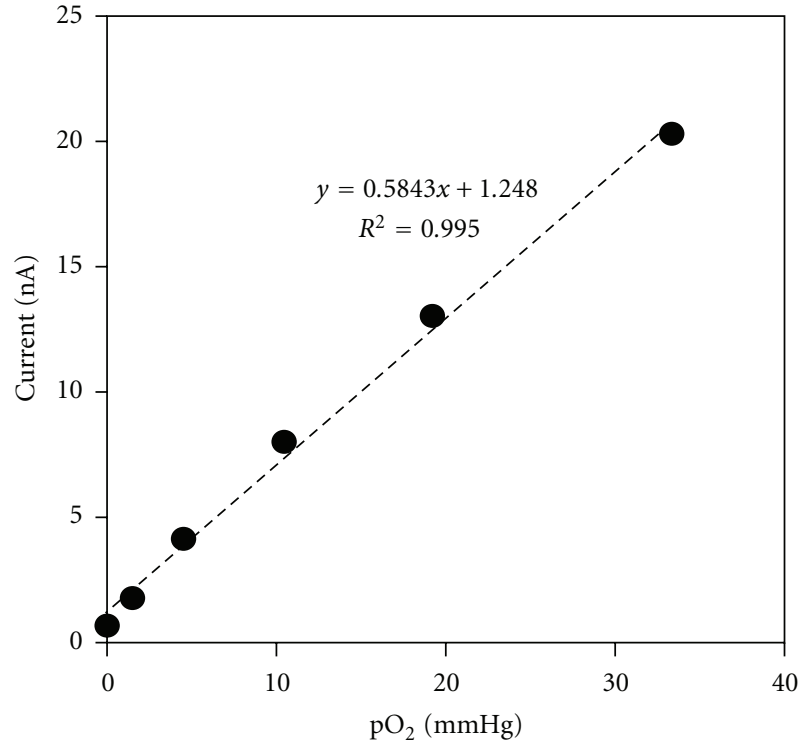

(b)

Figure 2: (a) A typical current response curve of an oxygen microsensor with the varied oxygen concentration. (b) Corresponding calibration curve in terms of $\mathrm{pO}_{2}$.

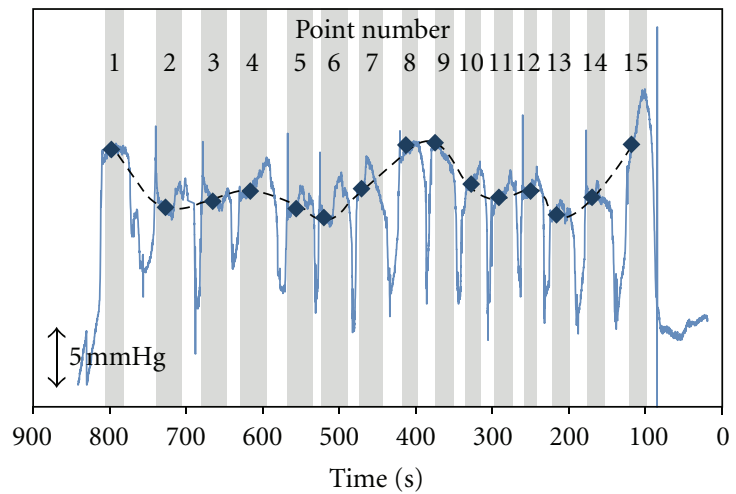

(a)

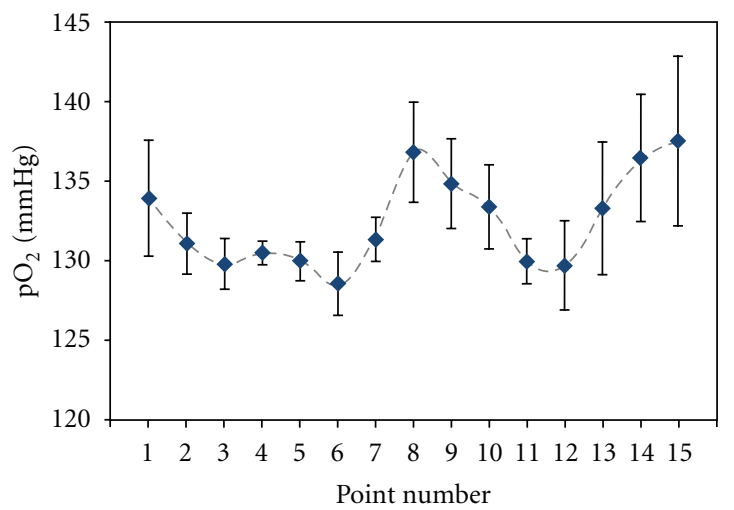

(b)

FIgURE 3: (a) A representative $\mathrm{pO}_{2}$ measurement along the wrist transverse crease as shown in Figure $1(\mathrm{a})$. The $\mathrm{pO}_{2}$ values were measured continuously at 15 different points with the one-dimensional sensor movement. Note that time increases to the left direction in $x$ axis, since the measurement was carried out with the sensor movement from point no. 15 to point no. 1 . The scattered symbols representing the pO $\mathrm{O}_{2}$ values measured at corresponding points are overlaid for clear presentation. (b) Averaged $\mathrm{pO}_{2}$ levels $(n=5)$ for 15 different points. The sensor's measurements at each point were averaged across five subjects. A paired $t$-test with a Bonferroni correction was conducted for six representative points (nos. 4, 6, 8, 10,14, and 15), and the $P$ values are summarized in Table 1.

Thus, the points exhibiting the $\mathrm{pO}_{2, \text { norm }}$ values less or greater than 1 represent the areas where the measured $\mathrm{pO}_{2}$ is lower or higher than the average, respectively. Each $\mathrm{pO}_{2 \text {,norm }}$ was obtained by averaging the two $\mathrm{pO}_{2 \text {,norm }}$ values obtained from two separate measurements at the corresponding same location for each subject. The average values of $25 \mathrm{pO}_{2}$ values were laid within a range of $130-140 \mathrm{mmHg}$ for five subjects.

Figure 4 is the color-coded contour plots of a typical two-dimensional oxygen measurement for one subject. For these contour plots, a linear change in $\mathrm{pO}_{2}$ was assumed between two adjacent points. As in the one-dimensional experiment, the $\mathrm{pO}_{2}$ values were varied depending on the locations, showing the heterogeneity of skin oxygen levels. The acupuncture point-high $\mathrm{pO}_{2}$ relationship is observed more clearly in the two-dimensional analysis. According to Eastern medicine, there are eight acupuncture points known in the wrist region for which the oxygen measurements were carried out: LU9 (Taiyuan) and LU8 (Jingqu) on the lung meridian; PC7 (Daling) and PC6 (Neiguan) on the pericardium meridian; and HT7 (Shenmen), HT6 (Yinxi), HT5 (Tongli), and HT4 (Lingdao) on the heart meridian. The locations of these acupuncture points are indicated in 


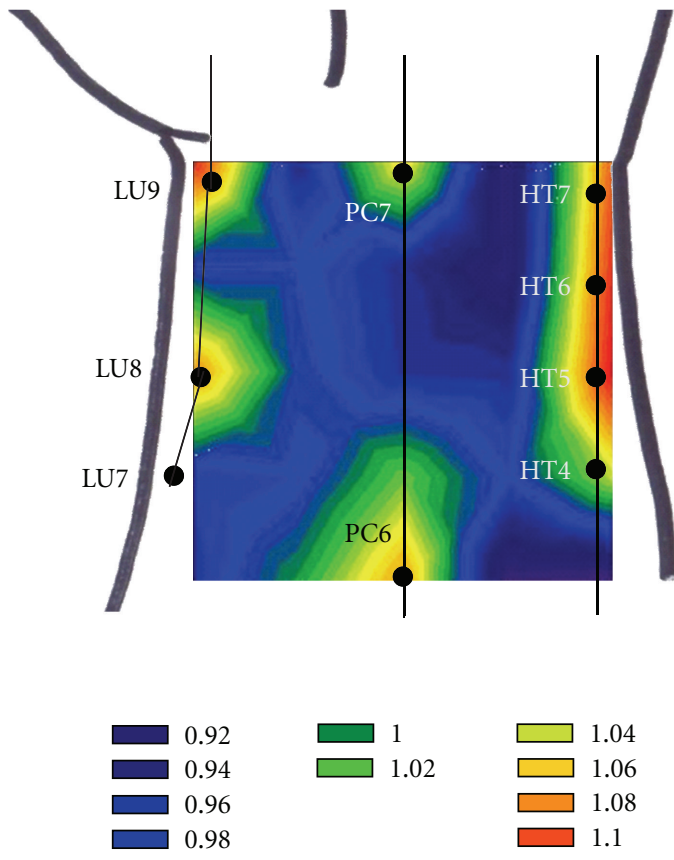

(a)

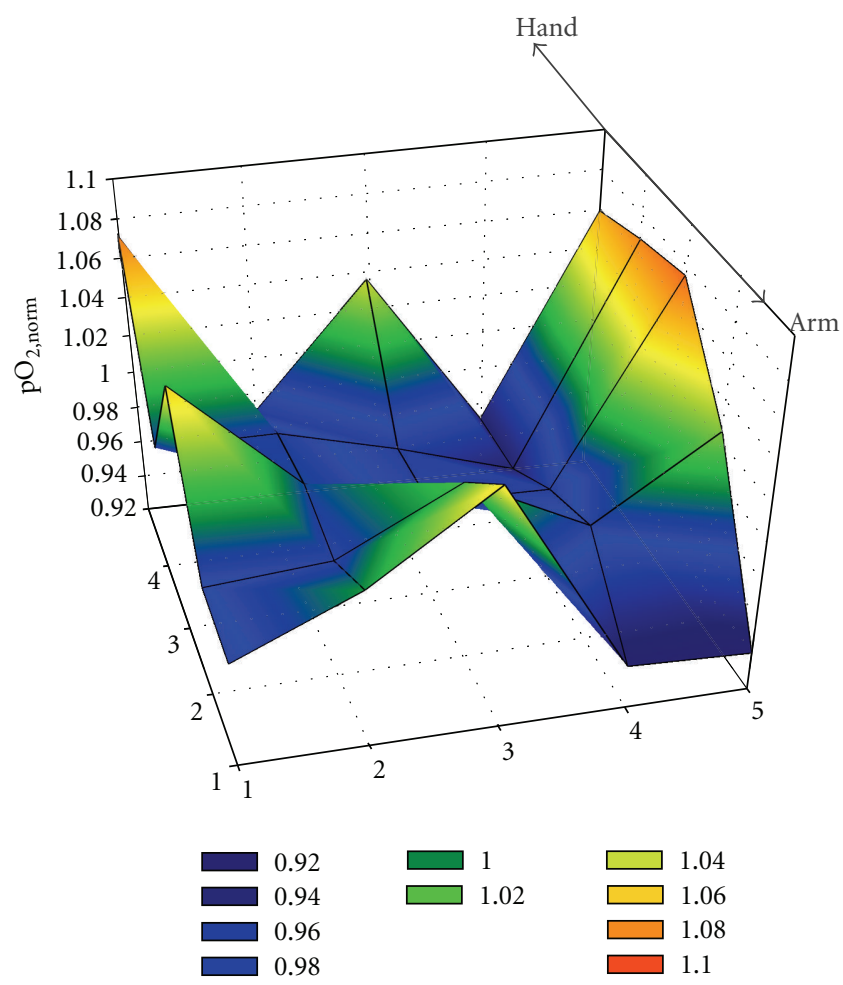

(b)

Figure 4: (a) 2-D and (b) 3-D illustration for the color-coded contour plots for a typical example of the two-dimensional oxygen measurement over the wrist skin. A linear change in the $\mathrm{pO}_{2 \text {,norm }}$ values was assumed between two adjacent points.

TABLe 1: Calculated $P$ values for the paired $t$-test $\left({ }^{*} P<0.05\right)$.

\begin{tabular}{lccccc}
\hline Point no. & P6 & P8 & P11 & P14 & P15 \\
\hline P5 & 0.0561 & $0.0181^{*}$ & 0.962 & $0.0369^{*}$ & $0.0488^{*}$ \\
P6 & & $0.0157^{*}$ & 0.361 & $0.0267^{*}$ & $0.0403^{*}$ \\
P8 & & & $0.0015^{*}$ & 0.856 & 0.677 \\
P11 & & & & $0.0165^{*}$ & $0.0149^{*}$ \\
P14 & & & & & 0.499 \\
\hline
\end{tabular}

Figure 4(a). In fact, the $\mathrm{pO}_{2 \text {,norm }}$ values are greater than 1 (i.e., higher $\mathrm{pO}_{2}$ level than the average) at the points close to the area where these acupuncture points are supposed to be present. Another typical example is shown in Figure 5. In this example, heterogeneous $\mathrm{pO}_{2}$ distribution over the wrist is also observed, obviously showing the strong correlation between the acupuncture point and high $\mathrm{pO}_{2}$ level. Similar $\mathrm{pO}_{2}$ distribution patterns were observed for overall five subjects.

As indicated in our previous report [14], the measured $\mathrm{pO}_{2}$ value shows a dependence on the distance between the sensor end plane and skin. In fact, the higher $\mathrm{pO}_{2}$ was observed when the sensor-skin separation was shorter. However, the $\mathrm{pO}_{2}$ variation induced by a slight difference in the sensor-to-skin separation during the experimental course was relatively small. In fact, $\mathrm{pO}_{2}$ variation $<1 \mathrm{mmHg}$ was observed from 10 separate measurements at the same point of one subject, supporting the reliability of the sensor movement/reposition procedure. Since the $\mathrm{pO}_{2}$ difference depending on the location is reasonably greater than the one induced by the sensor vertical positioning, the observed heterogeneous $\mathrm{pO}_{2}$ distribution can be considered to be valid.

The reason for the relatively higher $\mathrm{pO}_{2}$ levels around acupuncture points is not clearly understood yet. One possible explanation is that the oxygen supply by capillary oxygen transport is greater, and the oxygen uptake from the atmosphere is lesser around the acupuncture points than the other area. The oxygen supply to skin was demonstrated as a balance between the oxygen transport by blood and uptake from the atmosphere by Stücker et al. [16, 17]. Therefore, large blood vessels or Primo-nodes/vessels would possibly exist underneath the skin acupuncture points. Primovascular system was first proposed to be corresponding to acupuncture points by Kim [18] and recently rediscovered as a new circulatory system by Seoul National University group [19]. Further research, such as $\mathrm{pO}_{2}$ analysis combined with anatomical study, needs to be performed to clarify the possible relationship between Primo-vascular system and acupuncture points.

Regarding the connection between gas and acupuncture points, higher $\mathrm{pO}_{2}$ at the acupuncture points was previously reported for rabbits while the $\mathrm{pO}_{2}$ was measured in the tissue $\sim 0.5 \mathrm{~cm}$ below four selected skin acupuncture points (ST36, ST37, CV16, and CV17) with the sensor insertion [20]. In addition, higher transcutaneous carbon dioxide emission at 

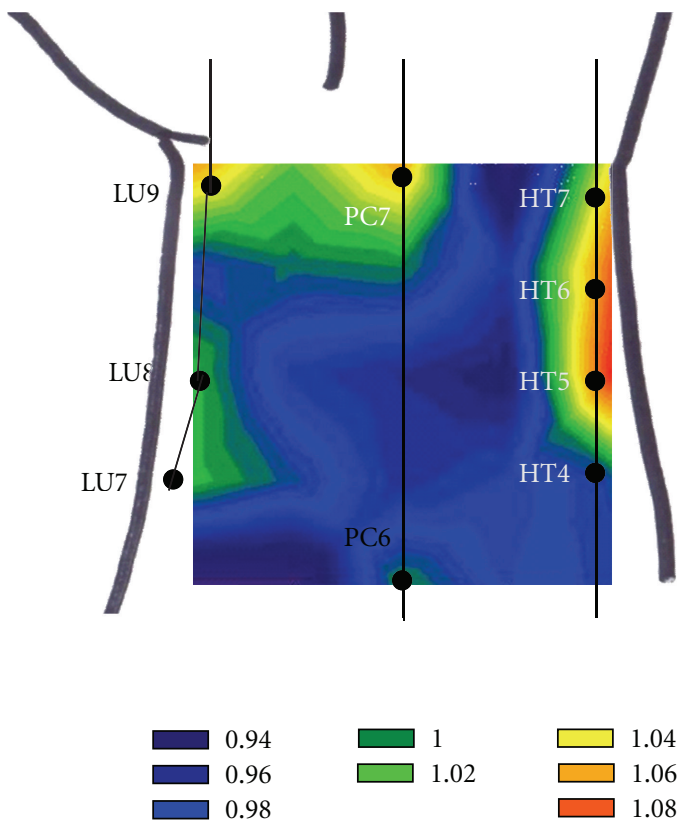

(a)

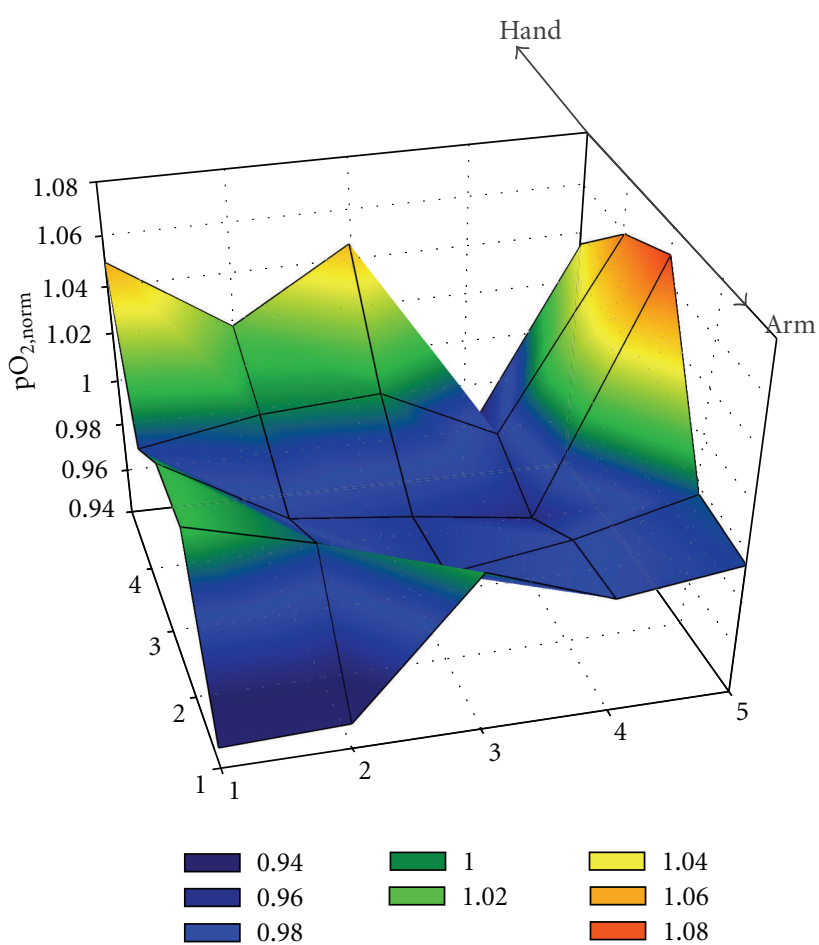

(b)

FIgURE 5: (a) 2-D and (b) 3-D illustration for the color-coded contour plots for another typical example of the two-dimensional oxygen measurement over the wrist skin. A linear change in the $\mathrm{pO}_{2, \text { norm }}$ values was assumed between two adjacent points.

12 acupuncture points on the pericardium meridian was reported compared with control points beside the meridian line [21]. Including these reports, the research regarding the acupuncture points and meridian has been conducted by comparing the characteristics of acupuncture points with that of control. Our current work provides clearer evidence on the strong $\mathrm{pO}_{2}$-acupuncture point correlation successfully by extending the analysis to the two-dimensional as well as noninvasive one. This investigation may verify the physical existence of the acupuncture points and provide helpful information to understand their physiological/biological functions believed in Eastern medicine for ages.

\section{Conclusions}

The skin surface $\mathrm{pO}_{2}$ levels measured for the anterior aspect of the left wrist varied depending on the locations in both the one-dimensional and two-dimensional analyses. The regions showing relatively higher $\mathrm{pO}_{2}$ levels compared to the other regions showed a strong correlation to the positions of acupuncture points for entire five subjects without exception. The higher $\mathrm{pO}_{2}$ values near the acupuncture points were observed with statistical significance. The used amperometric oxygen microsensor to monitor $\mathrm{pO}_{2}$ provided high sensitivity and small disk sensing area (diameter = $25 \mu \mathrm{m}$ ) which are sufficient to detect the $\mathrm{pO}_{2}$ variation as a function of location. Current study provides direct and scientific evidence on the physical existence of acupuncture points and may contribute to understand their possible biological/physiological functions.

\section{Authors' Contribution}

M. Hong, S. S. Park, and Y. Ha contributed equally to this work.

\section{Acknowledgment}

This research was supported by the Converging Research Center Program funded by the Ministry of Education, Science and Technology (2011K000813).

\section{References}

[1] M. W. Beal, "Acupuncture and oriental body work: traditional and biomedical concepts in holistic care: history and basic concepts," Holistic Nursing Practice, vol. 14, no. 3, pp. 69-78, 2000.

[2] National Institutes of Health, "Acupuncture: NIH consensus statement," U.S. Department of Health and Human Services, vol. 15, pp. 1-34, 1997.

[3] H. C. Dung, "Anatomical features contributing to the formation of acupuncture points," American Journal of Acupuncture, vol. 12, no. 2, pp. 139-143, 1984.

[4] J. Bossy, "Morphological data concerning the acupuncture points and channel network," Acupuncture and Electro-Therapeutics Research, vol. 9, no. 2, pp. 79-106, 1984. 
[5] M. Ciszek, J. Szopinski, and V. Skrzypulec, "Investigations of morphological structure of acupuncture points and meridians," Journal of Traditional Chinese Medicine, vol. 5, no. 4, pp. 289-292, 1985.

[6] H. Heine, "Anatomical structure of acupoints," Journal of Traditional Chinese Medicine, vol. 8, no. 3, pp. 207-212, 1988.

[7] H. Heine, "Functional anatomy of traditional Chinese acupuncture points," Acta Anatomica, vol. 152, pp. 293-297, 1995.

[8] A. Li, J. Zhang, and Y. Xie, "Human acupuncture points mapped in rats are associated with excitable muscle/skinnerve complexes with enriched nerve endings," Brain Research, vol. 1012, no. 1-2, pp. 154-159, 2004.

[9] S. Pearson, A. P. Colbert, J. McNames, M. Baumgartner, and R. Hammerschlag, "Electrical skin impedance at acupuncture points," The Journal of Alternative and Complementary Medicine, vol. 13, no. 4, pp. 409-418, 2007.

[10] A. C. Ahn, A. P. Colbert, B. J. Anderson et al., "Electrical properties of acupuncture points and meridians: a systematic review," Bioelectromagnetics, vol. 29, no. 4, pp. 245-256, 2008.

[11] A. C. Ahn, M. Park, J. R. Shaw, C. A. McManus, T. J. Kaptchuk, and H. M. Langevin, "Electrical impedance of acupuncture meridians: the relevance of subcutaneous collagenous bands," Plos ONE, vol. 5, no. 7, Article ID e11907, 2010.

[12] S. X. Ma, "Enhanced nitric oxide concentrations and expression of nitric oxide synthase in acupuncture points/meridians," Journal of Alternative and Complementary Medicine, vol. 9, no. 2, pp. 207-215, 2003.

[13] F. M. Faraci, "Role of nitric oxide in regulation of basilar artery tone in vivo," American Journal of Physiology, vol. 259, no. 4, pp. H1216-H1221, 1990.

[14] Y. Lee, G. Lee, S. S. Park, A. Jang, and G. J. Jhon, "Heterogeneity of skin oxygen density distribution: relation to location of acupuncture points," Journal of Acupuncture and Meridian Studies, vol. 2, no. 4, pp. 269-272, 2009.

[15] Y. Lee, B. K. Oh, and M. E. Meyerhoff, "Improved planar amperometric nitric oxide sensor based on platinized platinum anode. 1. Experimental results and theory when applied for monitoring NO release from diazeniumdiolate-doped polymeric films," Analytical Chemistry, vol. 76, no. 3, pp. 536$544,2004$.

[16] M. Stücker, P. Altmeyer, A. Struk et al., "The transepidermal oxygen flux from the environment is in balance with the capillary oxygen supply," Journal of Investigative Dermatology, vol. 114, no. 3, pp. 533-540, 2000.

[17] M. Stücker, A. Struk, P. Altmeyer, M. Herde, H. Baumgärtl, and D. W. Lübbers, "The cutaneous uptake of atmospheric oxygen contributes significantly to the oxygen supply of human dermis and epidermis," The Journal of Physiology, vol. 538, no. 3, pp. 985-994, 2002.

[18] B. H. Kim, "On the Kyungrak system," Journal of Academic Medical Science DPR Korea, vol. 90, pp. 1-35, 1963.

[19] K. S. Soh, "Bonghan circulatory system as an extension of acupuncture meridians," Journal of Acupuncture and Meridian Studies, vol. 2, no. 2, pp. 93-106, 2009.

[20] W. Xu, W. Ma, K. Li et al., "A needle-electrochemical microsensor for in vivo measurement of the partial pressure of oxygen in acupuncture points," Sensors and Actuators, vol. 86, no. 2-3, pp. 174-179, 2002.

[21] W. B. Zhang, Y. Y. Tian, Z. X. Zhu, and R. M. Xu, "The distribution of transcutaneous $\mathrm{CO}_{2}$ emission and correlation with the points along the pericardium meridian," Journal of Acupuncture and Meridian Studies, vol. 2, no. 3, pp. 197-201, 2009. 


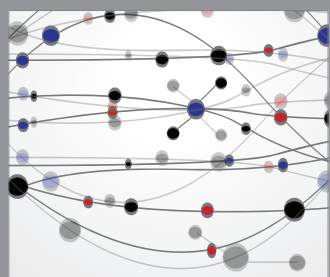

The Scientific World Journal
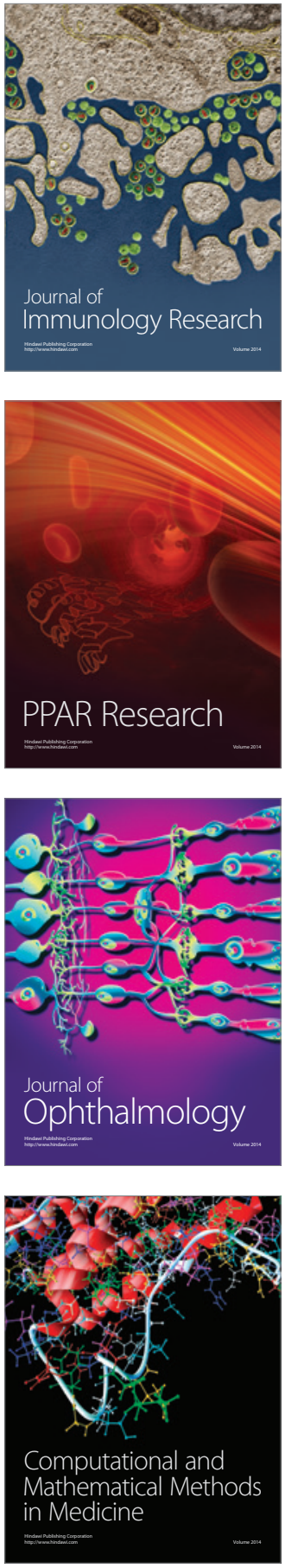

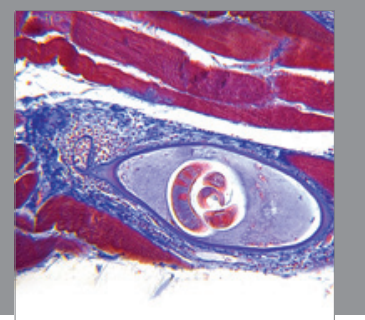

Gastroenterology

Research and Practice
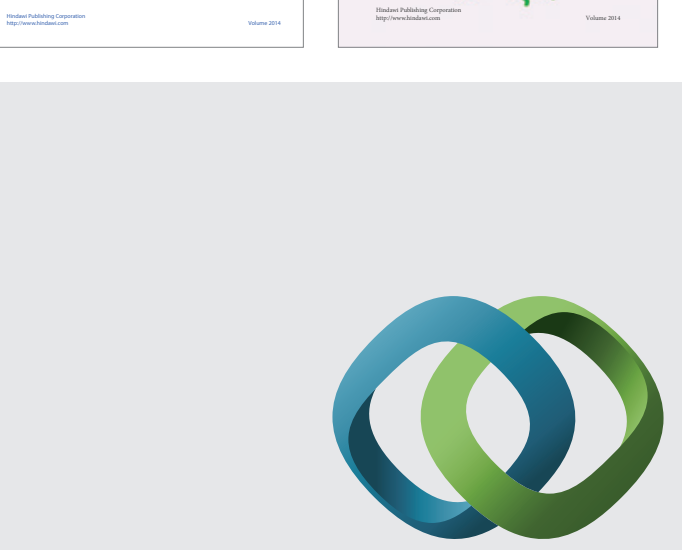

\section{Hindawi}

Submit your manuscripts at

http://www.hindawi.com
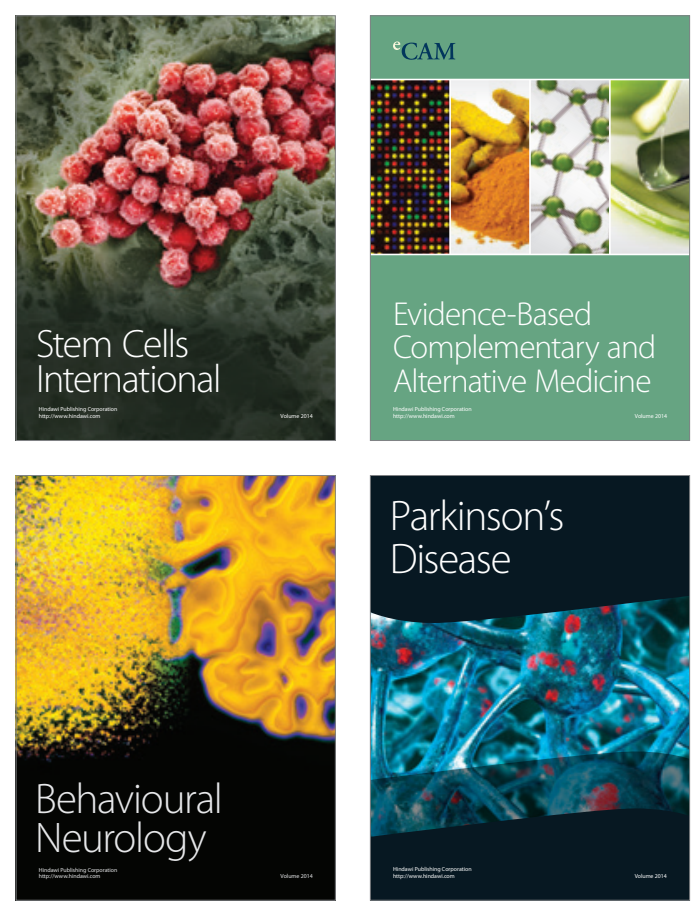

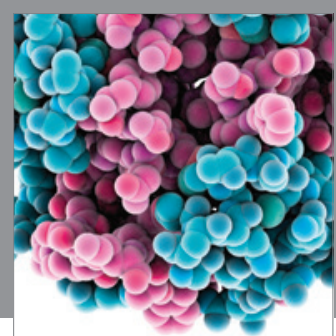

Journal of
Diabetes Research

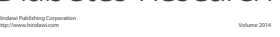

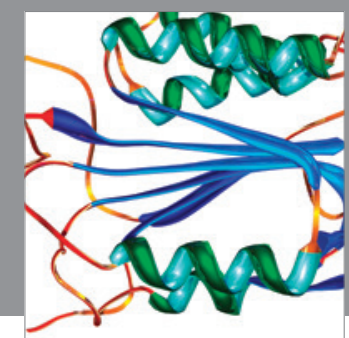

Disease Markers
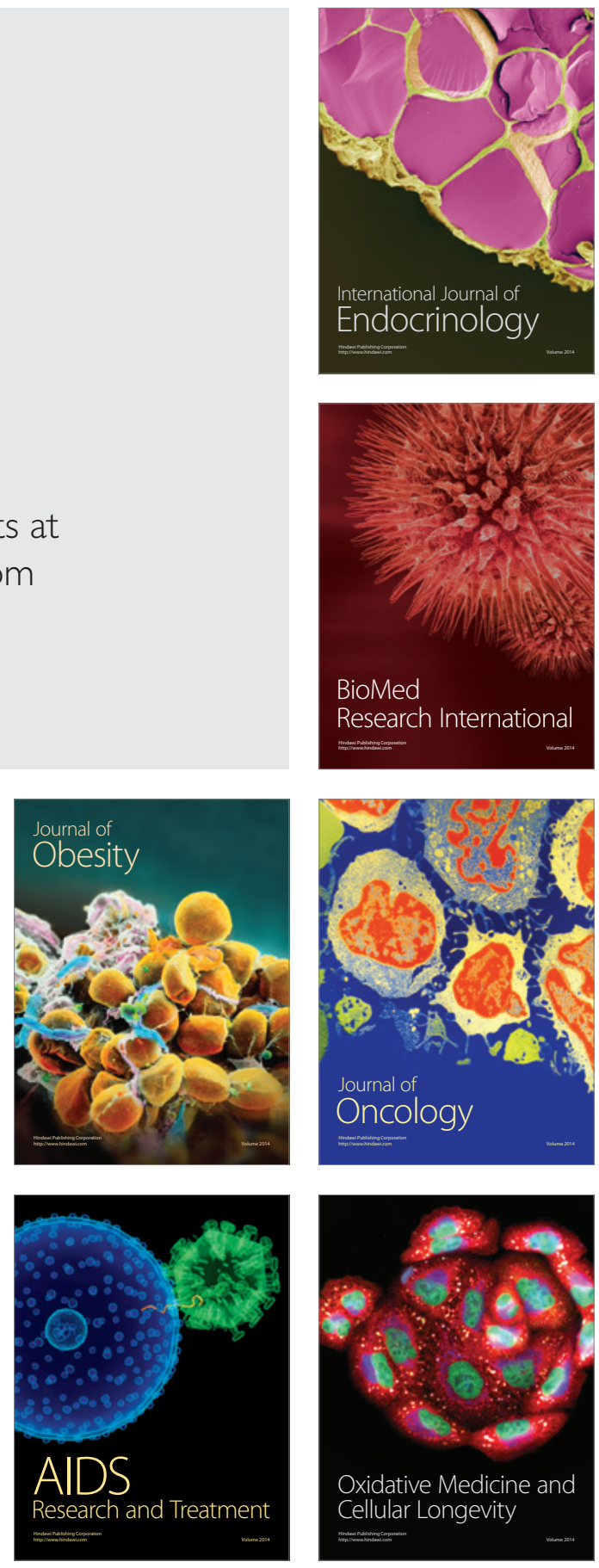